\title{
Strates
}

STRATES Matériaux pour la recherche en sciences sociales

$11 \mid 2004$

Jeune recherche, la vitalité d'un laboratoire

\section{Figures et paysages de la routine}

\section{Florent Hébert}

\section{OpenEdition}

Journals

Édition électronique

URL : http://journals.openedition.org/strates/503

DOI : $10.4000 /$ strates.503

ISSN : $1777-5442$

Éditeur

Laboratoire Ladyss

Édition imprimée

Date de publication : 1 janvier 2004

ISSN : 0768-8067

Référence électronique

Florent Hébert, "Figures et paysages de la routine », Strates [En ligne], 11 | 2004, mis en ligne le 15 février 2005, consulté le 08 septembre 2020. URL : http://journals.openedition.org/strates/503 ; DOI : https://doi.org/10.4000/strates.503

Ce document a été généré automatiquement le 8 septembre 2020

Tous droits réservés 


\title{
Figures et paysages de la routine
}

\author{
Florent Hébert
}

1 Quand je me suis intéressé aux pratiques et aux espaces qui se développent aujourd'hui grâce à (ou à cause de) la mobilité routière, ce qui m'a d'abord frappé, c'est la grande monotonie des trajets qui en résulte, et qu'accentue encore la banalisation des zones résidentielles, industrielles ou commerciales ; mais c'est surtout en fait la façon dont les populations arrivent à s'en accommoder.

2 Le travail que j'entreprends portera essentiellement sur les représentations locales de l'espace routier, et plus précisément sur la façon dont elles se mettent en place. En effet, les itinéraires quotidiens participent de façon essentielle à la définition géographique des localités : pour une population, les habitudes spatiales et temporelles que leur impose la voirie font en quelque sorte "partie du paysage", sans que l'on mesure bien l'influence exacte de l'aménagement des routes sur cette reconnaissance. Il s'agit pour moi de rendre compte de l'existence ou non de «mécanismes » routiers, par lesquels se développerait, de façon individuelle et collective, le sentiment d'une appartenance locale à un milieu. Ainsi, à travers l'étude de plusieurs situations, on peut identifier certains modèles de représentation de l'espace routier, connaître les conditions de leur apparition et mieux en tenir compte par la suite, par exemple lors d'un projet impliquant la mise en œuvre locale d'un réseau viaire. En effet, les débats publics locaux portent souvent sur les rapports entre l'espace de la circulation et d'autres, plus « résidentiels »; les conflits sont fréquents, qui opposent tout à la fois les collectivités et les associations, les opérateurs du réseau routier, les riverains ou les usagers, etc.

3 S'il semble aujourd'hui difficile de s'entendre sur l'importance spatiale relative à donner aux supports de la circulation automobile, c'est parce qu'on stigmatise, à mon sens, l'idée de la route "frontière" ou de la route "cicatrice", en se demandant d'emblée comment résoudre ce problème. Il me paraît plus important de chercher à savoir si s'établissent, par ailleurs, des "correspondances", des complémentarités, entre les lieux routiers et les lieux résidentiels; et si oui, dans quelles circonstances elles s'effectuent. Je prendrai ici l'exemple du lotissement de maisons individuelles, qui n'existerait pas sans l'apport de la mobilité routière; ce modèle d'habiter soulève 
plusieurs interrogations quant aux représentations de l'espace qui y sont mises en jeu, par le rapport exemplaire entre standardisation et localité qu'il symbolise; j'appliquerai ensuite ce questionnement au domaine routier qui lui est complémentaire. Il s'agira ainsi d'esquisser deux grands principes de structuration de l'espace qui semblent, aujourd'hui, communs à la mobilité et à l'habitat, et dont le champ d'application, par l'usage de l'automobile, s'étend désormais au paysage: la standardisation formelle et fonctionnelle des lieux, et le développement de temporalités circulaires, routinières, liées aux pratiques qui s'y déroulent.

Route et architecture : la nécessité d'un lien géographique

4 Lors de mon travail de fin d'études en architecture (Hébert, 2000), je me suis retrouvé confronté à l'espace, problématique en France, du lotissement contemporain, que symbolise plus particulièrement la maison dite « individuelle». Cet habitat me semblait procéder d'une logique spatiale que ma propre expérience de la maison standardisée me poussait alors à comprendre, mais à laquelle mes études ne m'avaient pas préparé. Les idées qui circulaient à la fin des années 80 paraissaient même contradictoires. D'une part, les dimensions culturelles que l'on prêtait au mode de vie des habitants de la maison individuelle semblaient inappropriées, car on les confondait le plus souvent avec celles de l'habitat pavillonnaire des Trente glorieuses ${ }^{1}$. D'autre part, chez les architectes, l'alternative au mitage du paysage et aux démembrements parcellaires des lotisseurs tournait invariablement soit autour de l'idée de la villa, soit autour de l'image du hameau rural (comme agglomération de maisons et de dépendances); ces modèles s'imposaient comme les seules références « acceptables " (c'est-à-dire traditionnelles) et esthétiquement correctes à l'espace de vie des habitants « individuels $»^{2}$. Or pendant ce temps, autour des petites villes, la construction des mêmes maisons banales et stéréotypées (car nous les connaissons tous) se répétait, sous la pression d'une demande relativement populaire. La dénomination d'habitat « individuel » (expression même de l'apparente fermeture de ses occupants à toute relation collective...) a peutêtre empêché de voir dans ces lotissements un espace public : ils sont pourtant le reflet direct et quasi-spontané d'un mode de vie partagé par toute une population, s'adaptant ainsi à un monde devenu plus "technique " dans ses modes de production, mais pas nécessairement plus savant dans ses rapports à l'environnement ${ }^{3}$.

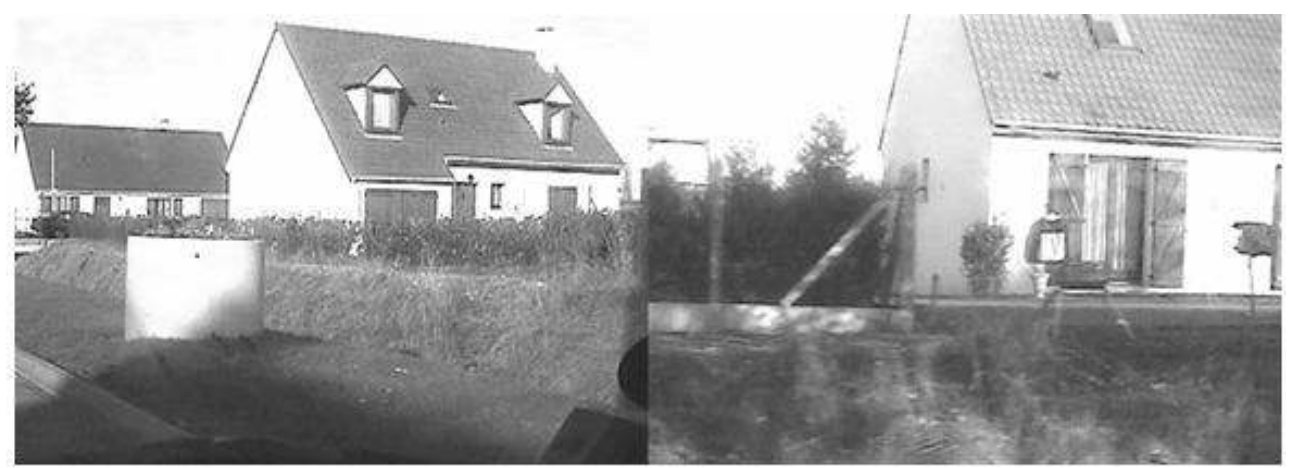

(c) Lotissement à Rosny-sur-Seine, Yvelines. Photo F. Hébert.

5 Le lotissement contemporain est en ce sens l'expression spatiale des (non) désirs de ses occupants ; ceci lui confère un aspect formel aisément identifiable : il est la réponse (ou l'absence de réponse) urbaine et architecturale à un mode d'habiter spécifique ; cela se traduit par la standardisation non seulement des maisons, mais aussi des jardins et des rapports à la nature qui peuvent s'y développer, sous la forme d'un mitage «aseptisé » 
apte à révéler une collectivité informelle. L'image que nous renvoient les lotissements vernaculaires contemporains est en ce sens paradoxale, donc remarquable: l'achat d'une parcelle de terrain semble exprimer la détermination de ses propriétaires à établir un rapport terrien à la nature, à un territoire. Mais visuellement, on observe assez peu de traces de l'expression de ce désir: les signes qui prouveraient une réelle appropriation singulière et physique (personnelle) du sol semblent absents du lotissement; ce qui se traduit par une certaine banalisation consensuelle de l'espace: thuyas, pelouse, automobile, balançoires vertes et autres meubles en pvc. Je ne parle pas seulement de l'aspect architectural des maisons, qui n'est ici que le résultat d'une technique de construction au moindre coût: en tant qu'objets de série, il semble admissible, car cela n'est pas nouveau, que les maisons puissent se ressembler (au même titre que les voitures), et qu'elles ne fassent pas l'objet de modifications spectaculaires (coûteuses) de la part de leurs propriétaires. Mais pourquoi en est-il de même quand il s'agit d'extérieurs privatifs, pourtant plus facilement malléables? Pourquoi là aussi cette ressemblance, cette redondance?

Plus encore que la maison, le jardin (le «terrain » comme on l'entend dire même longtemps après son " aménagement ») semble finalement exprimer, par son apparente vacuité, comme un besoin d'anonymat de ses habitants, la revendication d'une certaine neutralité territoriale ; celle-ci renforce le caractère "isotopique" des parcelles : la maison individuelle entre ainsi en contradiction flagrante avec les modèles de la villa ou du hameau qui peuvent, même encore aujourd'hui, être rapportés à des traditions architecturales (rurales ou urbaines) représentatives de l'identité de leurs occupants, et de leurs rapports à la collectivité et à la nature. On entend pourtant dire que si les accédants à la propriété choisissent de préférence la maison individuelle, c'est pour se mettre au vert. Cette soi-disant intention de retour à la nature, invoquée parfois et prêtée aux « individuels », peut, au regard de l'environnement de certains lotissements, faire sourire sinon paraitre méprisante, quand on prétend en faire la seule cause de la grande popularité de cette forme d'habitat. S'il existe un désir de présence organique, ici, ce serait peut-être plus dans la structure même du milieu que dans ses formes; le besoin de nature, comme partout, est présent, même s'il semble difficile parfois de le satisfaire directement ; le réseau viaire, par sa technicité actuelle, est peut-être devenu de ce point de vue indispensable : la manière dont la signalisation routière autorégule les circulations ne lui confère-t-il pas à la longue un statut plus naturel qu'artificiel ? Tandis que les représentations médiatiques du monde se succèdent, la route n'est-elle pas aujourd'hui assimilée à un milieu tellurique, fait pour durer, sur lequel se déploie toute une vie, au sens propre comme au figuré ${ }^{4}$ ?

7 Ce qui me paraît intéressant dans les lotissements contemporains, c'est leur interdépendance avec l'espace viaire, et le fait que la mobilité soit ainsi devenue l'expression d'un mode de vie, tout autant que l'habitat. Les traces singulières de pratiques territoriales domestiques ne semblent plus seulement se rapporter ici à des références spatiales (qui étaient celles des pavillonnaires) mais bien plus à des références temporelles, routines et habitudes qui ont trait à la vie de tous les jours, en train de se faire, et à leur développement. Contrairement à leurs prédécesseurs pavillonnaires, les habitants de la maison individuelle n'investissent visiblement pas beaucoup de temps à "produire » de l'espace, à domestiquer (par des interventions collectives ou individuelles) des lieux ; ils chercheraient plutôt à les reproduire, à les banaliser, à les rendre polyvalents pour mieux les asservir à leurs rythmes familiaux. Ce 
qui prime pour ces nouveaux habitants serait le simple déroulement de leur journée, c'est-à-dire le parcours et l'enchaînement des lieux où ils doivent se rendre, ou bien qu'ils doivent traverser (maison ; école ; supermarché ; carrefours ; etc.) ; l'exemple du lotissement (par ses répétitions localisées tant dans l'espace que dans le temps) me sert en quelque sorte d'hypothèse générale, car sa population semble mettre en place des itinéraires qui sont représentatifs d'un rapport singulier à un environnement (que ces liens «figurés " soient réels ou idéalisés, individuels ou collectifs, mais toujours entretenus jour après jour). Les habitants individuels savent rendre l'espace local. Ils structurent ainsi leur espace-temps, à travers sa récurrence, à défaut de pouvoir agir concrètement sur les lieux eux-mêmes comme sur leur rythme de vie. La circularité des emplois du temps domestiques, qui associent les lieux publics et privés suivant des routines temporelles adaptées, sont souvent les seules "preuves» tangibles de cette localité apparemment paradoxale du lotissement.

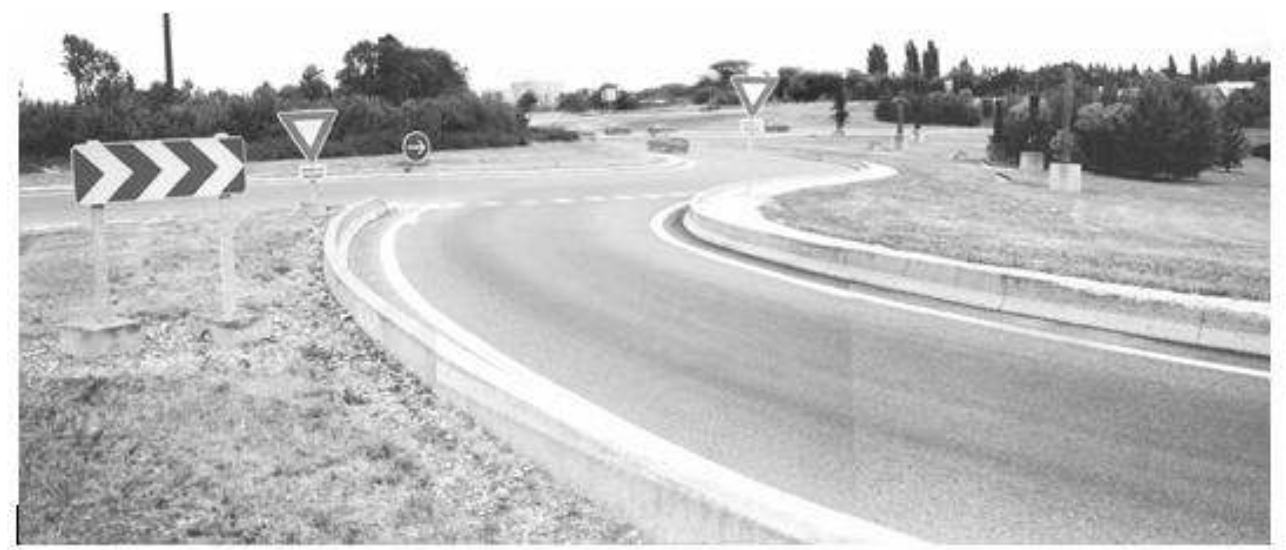

(C) Giratoire près de Dreux, Eure-et-Loire. Photo F. Hébert.

Dans un premier temps (celui du doctorat), je veux m'attacher à valider l'hypothèse que les circuits routiers, par leur parcours comme par leur présence, occupent une place importante dans nos cultures de l'espace. S'il paraît opportun de considérer le problème de l'habitat contemporain sous l'angle de la mobilité, il me semble réciproquement tout aussi juste désormais d'étudier les lieux de la mobilité routière comme étant indispensables à la structuration des représentations territoriales. Il me faut montrer qu'il existe un espace routier qui associe les pratiques routières avec les lieux et les représentations de celles-ci ; l'étude des aménagements peut ensuite révéler la nature locale des liens paysagers qui unissent, quelque part, « la route à la maison ». Et si la fréquentation des circuits routiers délimite des pratiques et des comportements locaux spécifiques, ce "bouclage " singulier des lieux quotidiens exprime, à mon sens, la spécificité culturelle des espaces de vie actuels; celle-ci se traduit par l'existence d'itinéraires routiniers, qui impliquent tout un système géographique de représentations quotidiennes, le mot représentation ayant ici une signification véritablement théâtrale; ces "représentations» privées sont-elles ainsi plus facilement observables dans l'espace routier qu'elles partagent, qu'à travers les lieux résidentiels qu'elles impliquent? Peut-être. Ma recherche ne portera pas explicitement sur les représentations elles-mêmes, mais plutôt sur la manière qu'offre la route à un individu ou à une collectivité de les systématiser: de mettre "en place " les lieux traversés, jusqu'à les mettre « en scène ». 
9 Si j'ai parlé de la maison individuelle, c'est aussi parce que la place si singulière qu'elle occupe actuellement dans notre pays me semble révélatrice d'une manière contemporaine de vivre le paysage : c'est-à-dire à la condition suffisante et nécessaire qu'un milieu puisse être parcouru et habité dans un vécu ordinaire ${ }^{5}$. Existe-t-il, par la mobilité, des représentations locales, dynamiques et routinières, qui puissent nous dévoiler l'existence de ces paysages ordinaires? Ceux-ci ne se rapporteraient plus seulement aux éléments naturels qui occupent bien sûr l'espace routier ou résidentiel, mais également aux éléments techniques dont le transport a besoin pour s'effectuer, et à leur naturalisation implicite. Si cette assimilation existe et s'avère partagée, c'est que la route constitue alors un espace public de nature plus géographique qu'urbaine, dépassant ainsi les reproches faits au lotissement: l'égocentrisme supposé de ses parcelles, et l'inertie sociale de ses collectivités...

Le paysage et la routine

10 Je voudrais vérifier l'hypothèse que l'itinéraire peut rendre le monde habitable : qu'il est à la fois mode d'expression, et possibilité offerte à un jeu collectif entre espace intérieur et espace extérieur, entre espace circulatoire et espace « cadastral ». La route, en fin de compte, ne représente-t-elle pas l'horizon de cette quête d'un temps ordinaire et d'un environnement consensuel, qui caractérise l'habitat des individuels? L'espace viaire «supporte » en effet beaucoup des routines de la migration alternée : par les parcours et les pratiques qu'il favorise, par les règles de comportement qu'il édicte... Les pratiques individuelles de déplacement forgent, par la répétition du circuit routier et par l'apprentissage d'une géographie partagée, des lieux emblématiques et familiers, sans avoir pour autant à les « domestiquer ", à les approprier 6 .

11 Aujourd'hui, à la banalisation de l'espace résidentiel répond désormais celle de l'espace routier : par la réplication de ses figures (carrefours, giratoires, rues...), par ses modèles de voirie, ses matériaux préconisés, etc. Cette homogénéisation rencontre-t-elle un consensus populaire, comme c'est le cas, d'une certaine façon, pour la maison individuelle? Elle semble être le résultat d'actions aujourd'hui déléguées aux seuls acteurs locaux, plus ou moins représentatifs de leur population. Peut-on dire que leurs choix en matière d'aménagement routier reflètent réellement une vision locale de l'espace routier? Quel rôle bon ou mauvais la puissance publique joue-t-elle aujourd'hui dans la façon d'aménager les routes, quand on sait que ce domaine lui est encore exclusivement réservé ? Les pratiques de déplacement automobile se mettent aujourd'hui en place dans des environnements marqués par les représentations routières du passé, et dont la valeur signalétique liée à la sécurité routière semble être le seul dénominateur commun. Les usages de l'espace routier sont de plus en plus codifiés, ce qui implique un certain mimétisme des itinéraires : mais à première vue seulement, car c'est par la répétition de ceux-ci que naît, malgré tout, le sentiment d'une appartenance géographique; des lieux deviennent ainsi plus représentatifs que d'autres, et notamment par la présence de certaines « figures ${ }^{7}$ » routières, reconnues par beaucoup.

12 Il me faut chercher à connaître, d'une personne à l'autre, si ces lieux participent à la fabrication d'un «paysage» de la répétition et de la routine. Il existera vraisemblablement une certaine difficulté à mettre ainsi en évidence la représentativité territoriale, locale et paysagère, d'un circuit routier (dans quelles dimensions l'observer ?). Je cherche à savoir si, par l'intermédiaire de celui-ci, il est possible pour une population restreinte de se structurer un paysage, par lequel 
« habiter » (à défaut pour elle de pouvoir intervenir, de façon matérielle, sur les lieux qu'elle fréquente). Ainsi, dans notre simple confrontation quotidienne aux lieux de la circulation, il existerait des relations durables méconnues à prendre en compte dans la formation des identités et des territoires. Il existe bien sûr des préjugés quant à l'espace routier et aux lieux qui lui sont «techniquement» asservis. Dans le meilleur des cas, ceux-ci sont supposés symboliquement neutres, du moins si on les rapproche de ce que l'anthropologue Marc Augé nomme des "non-lieux ${ }^{8}$ ». Il faudra donc vérifier ces hypothèses : l'espace routier se trouve polarisé entre des lieux porteurs d'identité et des non-lieux porteurs d'anonymat. L'apprentissage (forcé) du circuit routier, sous la condition de la répétition quotidienne, implique une dépendance à la route, et celle-ci participe à l'élaboration de rapports territoriaux forts. La routine transformerait-t-elle le non-lieu en lieu ? L'espace routier semble rencontrer à la fois l'un et l'autre, dont l'habitant, captif des circonstances, se sert pour définir ses propres rapports paysagers avec le monde. Si la routine véhicule, malgré toute sa banalité et sa monotonie, du sens, c'est à mon avis parce qu'elle s'effectue en présence des non-lieux : les non-lieux et leur non-sens, par leur "disponibilité », offrent l'occasion d'une réinvention possible de «cosmologies » locales. Parler de l'espace routier, c'est donc parler d'abord de son équipement technique, et des figures de paysage imprévues qu'il génère: les carrefours, par leurs aménagements successifs et leur singularité géographique, paraissent être les archétypes de ce paysage de la routine routière.

Il me faut vérifier toutes ces hypothèses auprès d'une population " routinière ", par le biais d'une enquête. Celle-ci portera sur les conditions d'une reconnaissance locale de la route en tant qu'espace, mais devra, dans le même temps, poser certaines questions relatives à la genèse structurelle des paysages routiniers. Il me sera ainsi peut-être possible de dégager une approche théorique de l'espace routier non plus seulement esthétique ou technique (comme ont pu s'y attacher les historiens ou les urbanistes) mais territoriale et culturelle. Cependant, il peut paraître périlleux, en l'absence de groupes sociaux réellement constitués ou observables, de tenter de "monographier » par exemple l'influence d'un élément du circuit routier comme un carrefour sur la vie de tous les jours, en me référant exclusivement à des "usages » et à des pratiques individuelles ou familiales; en effet, quelles conclusions tirer du discours de personnes aussi différentes qu'un chauffeur de bus, un enfant utilisant son vélo pour se rendre à l'école, une mère faisant ses courses au supermarché, et ainsi de suite?

14 Si je pose l'hypothèse que les itinéraires structurent les représentations locales que produit l'espace routier, je dois montrer, par l'analyse des entretiens, deux choses: qu'il existe effectivement un véritable paradigme routier dans notre façon d'appréhender le monde; et que celui-ci est la conséquence directe de la métamorphose des routes, et non pas, par exemple, de l'évolution des moyens de transport, comme il est d'usage de le dire (une fois descendu de voiture, on n'oublie pas ce lien routier qui nous rattache au reste du monde, mais au contraire, c'est à ce moment qu'il nous est le plus nécessaire, le plus intime).

Or il existe une période charnière (un changement de paradigme justement) commune à beaucoup d'entre nous, et pendant laquelle nos représentations routières, mises en place par les itinéraires que nous fréquentons, ont été bouleversées : c'est lorsque nous avons appris à conduire, et à déchiffrer le code de la route. C'est pourquoi il me paraît plus pertinent d'interroger la population des «auto-écoliers» et des jeunes conducteurs, plutôt que celle des conducteurs plus confirmés. Par les nouvelles 
habitudes et pratiques qu'il instaure, le passage du permis de conduire agit en effet comme un révélateur de nos "anciens" modèles, ceux d'une population plus " captive ». Mais s'il s'agit de mettre en évidence les liens qui existent entre espace routier et espace résidentiel, peu importe. Dans les discours, il sera peut-être envisageable de dégager des similitudes entre les différentes expériences d'un même parcours ; mais le peu d'expérience des conducteurs ne permettra vraisemblablement pas d'établir alors de lien direct entre conduite et paysage ; le fait de conduire servira ici en fait de catalyseur au discours sur le passé proche d'un vécu de l'espace routier, tant il est vrai qu'il n'est pas nécessaire de savoir conduire pour être confronté d'une manière ou d'une autre à la route ${ }^{9} .$.

L'aménagement routier est-il le vecteur d'un nouveau mode d'existence du paysage ? Il s'agirait alors de prolonger le terme au-delà de son sens commun : ni image, ni même ensemble d'images, mais développement d'une représentation possible, «itinérante ", d'un réseau de lieux, dans le temps et dans l'espace. L'automobile permet ainsi d'augmenter la couverture géographique quotidienne de chacun à l'échelle même des paysages, tels qu'on les conçoit traditionnellement. Depuis l'après-guerre, son utilisation a considérablement modelé l'espace routier. Cette évolution technique a changé la nature des liens qui raccrochent les lieux familiers des espaces « résidentiels » aux " points-clefs ${ }^{10}$ » du réseau viaire; ceux-ci ne se distinguent plus seulement par des singularités géographiques, liées à la topologie des réseaux, mais ils possèdent désormais des singularités techniques et typologiques qui dépendent directement de l'évolution des moyens de transport. Or l'importance prise aujourd'hui par les boucles de déplacement fait que ces mêmes points-clefs routiers font correspondre chaque jour, concrètement et abstraitement, les corps et les lieux, symbolisant ainsi des temporalités locales; ils sont donc les figures singulières de notre relation contemporaine à la route, et définissent, par leur présence ambivalente, ce que j'appelle un paysage de la routine.

Enjeux et conclusion

17 La routine est-elle à considérer comme un mode de développement privilégié de nos médiations, de nos alliances avec l'espace de la mobilité et de ses lieux routiers? Il conviendra de s'interroger sur la nécessité d'en tenir compte dans tout aménagement du réseau viaire : car celui-ci, même dans ses parties les plus anodines, peut s'avérer porteur d'un sens géographique local, qui échappe pour l'instant à toute analyse technique ou même esthétique. Réciproquement, dans la colonisation de nouveaux territoires urbains par la voirie (ronds-points giratoires, boulevards urbains...), on gagnerait certainement à comprendre que les lieux de la circulation ne sont pas de simples outils de desserte ou de régulation du trafic, qui peuvent être impunément camouflés ou décorés; ils représentent ce par quoi le sentiment d'une appartenance géographique à un territoire peut encore se structurer et se développer, de façon complexe, mais suivant toutefois des modes de structuration relativement simples. Ce n'est donc pas parce que cette structuration s'effectue dans un espace routier déjà constitué qu'il faut se dispenser d'intervenir en son nom... Bien au contraire, l'hégémonie de la puissance publique dans ce domaine est à remettre en question au profit de la connaissance locale que les populations peuvent lui apporter ; à la condition toutefois de réétudier, sous l'angle de la routine et de l'habitude, les grands principes de composition des modèles routiers établis jusqu'à présent ${ }^{11}$. 
Car la routine routière, comme habitude, met en boucle les territoires du quotidien dans une sorte d'hypnose technique que la sophistication croissante des aménagements viaires entretient. Cette " transe pendulaire " semble même renouer avec une certaine forme de rite territorial ; on est alors très proche de cet " espace réticulé ", mythique, qu'était parti étudier le géographe Joël Bonnemaison dans les îles Mélanésiennes :

Dans le territoire réticulaire, le lieu focal n'est plus le lieu central, mais le lieu fondateur. Le territoire ne s'organise pas selon le modèle du polygone centralisé, ou selon un modèle de type centre-périphérie, mais sur une configuration de type nexus. Du lieu fondateur surgit un espace-route où se transmettent, par bonds successifs, d'une maille à l'autre, les messages, les relations politiques et les rites d'alliance ${ }^{12}$.

\section{BIBLIOGRAPHIE}

Augé M., 1992, Non-lieux : introduction à une anthropologie de la surmodernité. Paris ; Seuil.

Bonnemaison J., 2000, La géographie culturelle. Cours de l'université Paris IV - Sorbonne 1994-1997. M. Lasseur, C. Thibauld (Éds). Paris, Éditions du CTHS, p. 20.

Hébert F., 2001, L'espace hybride des carrefours. Paysages. Mémoire de DEA (A. Roger, dir.). École d'architecture Paris La Villette - Université Paris 1, DEA Jardins paysages territoires.

Hébert F., 2000, Anti-déplacement. Mémoire de fin d'études (C. Girard \& F. Devaulchier, dir.) École d'Architecture Paris Villemin (Malaquais).

Jackson B., 2000, Le Visiteur, no 5, printemps. Paris ; Société française des architectes.

Lassus B., 1977. Jardins imaginaires. Paris ; Presses de la Connaissance, diffusion Weber.

Raymond H., Haumont N., Raymond M. G., Haumont A., 1966, L'Habitat pavillonnaire. Paris ; Institut de sociologie urbaine, Centre de Recherche d'Urbanisme.

Simondon G., 1958, Du mode d'existence des objets techniques, Paris, Aubier, éd. Montaigne.

\section{NOTES}

1.Les pratiques spatiales liées aux pavillons avaient été abordées par certains sociologues (Raymond, Haumont, Raymond, Haumont, 1966) et partiellement par Bernard Lassus dans son travail sur les « habitants-paysagistes » (Lassus, 1977). 2.Puisqu'on parle d'habitat individuel.

3.Les lotissements pavillonnaires d'après-guerre, et surtout ceux qui furent construits en collectivités (les "Castors ») nous paraissent ainsi aujourd'hui être issus d'un modèle plus « artisanal » de l'espace résidentiel. Mais on y trouve la même adéquation sociale et «non-savante » d'une population à son habitat, ce qui permet, d'après moi, de qualifier ces lotissements comme leurs successeurs d'habitat " vernaculaire ». 4.Cette possible « identification » des dispositifs routiers à des entités naturelles sera à prendre en compte lors de la mise au point d'une technique d'enquête. 
5.C'est notamment la position que défendait l'auteur américain J. B. Jackson (2000). À propos de "paysage ", il faut donc bien faire la distinction entre l'expérience quasitouristique, quasi-mystique des grands paysages naturels, et celle du paysage ordinaire ; celui-ci joue plutôt ici le rôle d'un système de " correspondances " géographiques, entre l'imagination et la réalité d'un espace qui entoure et que l'on parcoure. Ces relations se construisent non par une disponibilité à la contemplation du monde, mais par une habitude à la vacance symbolique des lieux : ce n'est pas l'observateur qui se trouve disponible, ce sont les lieux eux-mêmes.

6.Il reste à montrer par exemple de quelles manières ces « habitants-passagers » quand ils imaginent ou se représentent leur environnement, partagent les figurations si locales et singulières de leur mobilité que sont les carrefours. J'ai pu élaborer certaines hypothèses lors d'un travail préparatoire (Hebert, 2001).

7.Le terme de " figure » reflète à la fois l'idée d'une dimension topologique, et celle d'une individualité. Il suggère aussi que le mouvement puisse être un jeu, une danse. 8.Suivant les écrits de Michel de Certeau, M. Augé redéfinit ainsi l'importance de ces " non-lieux » de la mobilité auxquels nous sommes confrontés, par ce qu'il appelle un « excès de modernité » de notre société : la modernité se caractériserait par la possibilité d'une coexistence, dans un même espace du déplacement, d'éléments mythiques et symboliques, avec des éléments techniques et fonctionnels a priori non porteurs de sens ; parcourir cet ensemble crée alors l'espace complexe de la mobilité contemporaine. Toutefois, il est dit que le non-lieu caractérise la rupture entre le voyageur et le " paysage » : ramenée à une certaine échelle spatiale et temporelle (routinière), on peut penser malgré tout que la fréquentation des non-lieux et leur apprentissage, sous la condition de la répétition quotidienne, ne peuvent pas être assimilés à un voyage. Mais l'auteur n'aborde pas la mobilité répétitive, qui induit des rapports d'habitude. Il considère pourtant la bipolarité « fuyante » des lieux (entre sens et non-sens), et le champ alors ouvert à une écriture palimpseste des identités. Pour M. Augé, la fréquentation des non-lieux n'apporterait rien d'autre que l'expérience de la solitude, et s'opposerait même à la construction de liens territoriaux (Augé, 1992, p. 101-110).

9.Méthode envisagée : un premier groupe d'entretiens sera mené dans des auto-écoles françaises, localisées en milieu tant urbain que rural. Ce test sera effectué lors d'une projection audiovisuelle par des réponses en temps réel à un questionnaire à choix multiples ; il s'agira pour les apprentis-conducteurs d'identifier des figures routières locales, au travers de situations censées amener des réactions différenciables (à la manière d'un test pour le code de la route). Un deuxième groupe d'entretiens, plus restreint, sera basé sur le principe de la " conduite accompagnée ", et consistera à faire décrire à des sujets volontaires du premier groupe les itinéraires qu'ils parcourent, et la façon dont ils les percevaient avant d'être au volant ; ces narrations seront conservées par enregistrement audio-visuel. Je n'ai pas encore décidé quels outils d'évaluation et d'analyse allaient être employés.

10.Suivant la terminologie de G. Simondon (1958).

11.Je ne peux ici qu'effleurer le sujet, mais chaque carrefour raconte par sa morphogenèse l'histoire d'une "répression " publique qui s'exerce sur la vitesse des véhicules, et donc sur cette forme de « liberté d'expression " populaire que représente la conduite automobile, au nom de la seule Sécurité Routière... Ce qui pose pour moi un problème dès lors que cette normalisation des rapports entre pouvoir et population touche ainsi, indirectement, à toute autre représentation routière et au libre choix de 
celle-ci, sans tenir compte par exemple de son éventuelle implication dans la reconnaissance de la spécificité locale d'un itinéraire.

12.Bonnemaison (2000, p. 20).

\section{RÉSUMÉS}

D'un bout à l'autre de la France, les lotissements contemporains de maisons individuelles constituent un nouvel espace résidentiel qui, pris du point de vue de sa représentation, reste encore relativement énigmatique. Il semble en effet que les populations concernées ne cherchent pas à exprimer une quelconque spécificité locale dans leurs relations territoriales (à travers par exemple l'aménagement spatial, privé ou public, de lieux symboliques). Par la mobilité (et notamment à travers les boucles de déplacement quotidiennes) se constituent des itinéraires routiniers, qui semblent tenir lieu de représentation effective, pour ces habitants « individuels ", de leur localité. Il apparaît donc que des correspondances géographiques puissent exister entre espace résidentiel et espace routier, et que le rôle de celui-ci soit devenu primordial dans la perception et la définition d'un environnement local.

In all parts of France, the contemporary housing estates constitute a new residential space which, taken from the point of view of its representation, remains relatively enigmatic. Indeed, its inhabitants do not seem to try to express any local specificity in relationship with the space they occupy. Such a specificity could express itself through the public or private development of symbolic places. Mobility - and above all commutations - creates routine circuits which seem to work for these "individual" inhabitants as the effective representation of their locality. It seems therefore that geographical correspondences may exist between residential spaces and road networks, and that the role of the latter may be crucial in the perception and definition of local environments.

\section{AUTEUR}

\section{FLORENT HÉBERT}

Architecte DPLG, Géographe, doctorant au Ladyss, université de Paris 1, fl.hebert@free.fr 